\title{
BULLYING NA ADOLESCÊNCIA: UM ESTUDO COM BRASILEIROS E PORTUGUESES
}

\author{
Wanderlei Abadio de Oliveira ${ }^{a}$ \\ Jorge Luiz da Silva ${ }^{a}$ \\ Julliane Messias Cordeiro Sampaio ${ }^{b}$ \\ Beatriz Oliveira Pereirac \\ Marta Angélica lossi Silva ${ }^{d}$
}

\begin{abstract}
Resumo
O bullying é reconhecido como um problema de saúde pública. Existem diferenças nas taxas de prevalência do fenômeno entre países, mas poucas discussões ampliam a compreensão sobre essa variação. Assim, este estudo objetivou identificar a prevalência e as características de vitimização por bullying entre estudantes brasileiros e portugueses de acordo com a idade, o sexo e o ano escolar. Para tanto, realizou-se um estudo transversal com 182 estudantes - sendo 107 brasileiros e 75 portugueses - que frequentavam o 5o e o 6으 ano escolar. Os dados foram coletados por meio de um questionário autoaplicável e analisados estatisticamente pelo Teste Exato de Fisher. Os resultados permitiram a identificação de alta prevalência de estudantes vítimas de bullying nos dois países, sendo uma quantidade maior de estudantes do sexo masculino. A agressão verbal foi a mais sofrida nos dois países e a maioria dos estudantes relataram a outras pessoas as agressões. Além disso, demonstrou-se a universalidade do fenômeno, presente em escolas de diferentes contextos socioculturais. Assim, concluiu-se que o bullying influência no desenvolvimento, na saúde e no processo ensino-aprendizagem de crianças e adolescentes em idade escolar.
\end{abstract}

Palavras-chave: Violência. Saúde escolar. Saúde pública.

\footnotetext{
Psicólogos. Doutores em Ciências. Ribeirão Preto, São Paulo, Brasil.

b Enfermeira. Doutora em Ciências. Docente do Centro Universitário do Distrito Federal. Brasília, Distrito Federal, Brasil.

c Educadora Física. Doutora em Estudos da Criança. Docente catedrática do Instituto de Educação da Universidade do Minho. Braga, Portugal.

d Enfermeira. Doutora em Enfermagem em Saúde Pública. Professora associada da Escola de Enfermagem de Ribeirão Preto da Universidade de São Paulo. Ribeirão Preto, São Paulo, Brasil.

Endereço para correspondência: Avenida dos Bandeirantes, número 3.900, Campus Universitário. Escola de Enfermagem de Ribeirão Preto da Universidade de São Paulo, sala 72. Monte Alegre, Ribeirão Preto, São Paulo, Brasil. CEP: 14040-902.E-mail: wanderleio@usp.br
} 
Revista Baiana de Saúde Pública

BULLYING DURING ADOLESCENCE: A STUDY WITH BRAZILIANS AND PORTUGUESE

\begin{abstract}
Bullying is known as a public health problem. There are differences in the prevalence rates of the phenomenon between countries, but few discussions expand the understanding of this variation. Thus, this study aimed to identify the prevalence and characteristics of bullying among Brazilian and Portuguese students according to age, to sex and school year. Therefore, a cross-sectional study was performed with a total of 182 students - being 107 Brazilian and 75 Portuguese students - attending the 5th and 6th grades. Data were collected through a self-applied questionnaire and statistically analyzed using Fisher's Exact test. The results allowed the identification of a high prevalence of victims in both countries while the majority was male. Verbal aggression was the most common type in both countries and most students reported the aggressions to other people. Besides, it was evidenced the universality of the phenomenon that is present in schools from different sociocultural contexts. Thus, it was possible to conclude that the bullying influences the development, the health and the teaching-learning process of school-aged children and adolescents.
\end{abstract}

Keywords: Violence. School health. Public health.

\title{
BULLYING EN LA ADOLESCENCIA: UN ESTUDIO BRASILEÑOS Y PORTUGUESES
}

\section{Resumen}

El bullying es reconocido como un problema de salud pública. Hay diferencias en las tasas de prevalencia del fenómeno entre países, pero pocas discusiones amplían la comprensión de esta variación. Así, este estudio objetivó identificar la prevalencia y las características de la victimización por bullying entre estudiantes brasileños y portugueses según la edad, el sexo y el año escolar. Para ello, fue realizado un estudio transversal que incluyó 182 estudiantes - 107 brasileños y 75 portugueses - del $5^{\circ}$ y $6^{0}$ año escolar. Los datos fueron recogidos por medio de un cuestionario autoaplicable y analizados estadísticamente mediante por la Prueba Exacta de Fisher. Los resultados permitieron la identificación de alta prevalencia de estudiantes víctimas de bullying en los dos países, con una mayor cantidad de estudiantes del sexo masculino. La agresión verbal fue la más sufrida en los dos países y la mayoría de los estudiantes relató la agresión a otras personas. Además, fue demostrada la universalidad del fenómeno, presente en escuelas de diferentes contextos socioculturales. Así, concluyóse 
que el bullying afecta el desarrollo, la salud y el proceso de enseñanza-aprendizaje de niños y adolescentes con edad escolar.

Palabras-clave: Violencia. Salud escolar. Salud pública.

\section{INTRODUÇÃO}

O bullying é uma forma de comportamento agressivo intencional, que se repete sistematicamente ao longo do tempo, contra uma pessoa que se sente impotente para defender-se ${ }^{1}$ das agressões. As consequências do envolvimento em situações dessa natureza podem ser graves e duradouras ao longo do tempo, incluindo problemas como ansiedade, baixa autoestima, depressão, insônia e falta às aulas, por exemplo. Esses aspectos influenciam negativamente as condições de saúde e de aprendizagem de crianças e adolescentes em idade escolar $^{2-3}$.

A literatura científica indica que o bullying ocorre em todo mundo, não se limitando a uma região ou aos aspectos socioeconômicos e culturais ${ }^{4}$. As taxas de ocorrência desse fenômeno variam entre os países entre 7\% a 43\% para vítimas e de 5\% a 44\% para os agressores $^{5}$. Os meninos, em geral, estão mais associados aos episódios desse tipo de violência ${ }^{6}$. Sua ocorrência aumenta progressivamente nos anos escolares, tendo picos nos momentos de transição entre um ciclo escolar e outro, bem como no início da adolescência ${ }^{1,7-8}$.

A abordagem bioecológica ${ }^{9}$ do bullying permite conhecer os perfis dos participantes, as relações hierárquicas, os aspectos de cultura social e institucional das escolas, e como esses diferentes elementos podem auxiliar ou prejudicar o desenvolvimento saudável de crianças e adolescentes em idade escolar ${ }^{10-12}$. Em linhas gerais, a Teoria Bioecológica do Desenvolvimento ${ }^{9}$, estruturada em sistemas, contém cinco componentes importantes: individual; microssistema (famílias e escolas); mesossistema (família e escola); exossistema (atividades profissionais dos pais); e macrossistema (sistema de crenças e cultura, políticas públicas) $)^{9}$. O desenvolvimento de uma pessoa é construído por meio de intercâmbios complexos entre seus aspectos biopsicológicos e as outras pessoas, objetos, instituições e ambientes ${ }^{13}$. Para este estudo são relevantes os dois primeiros componentes, uma vez que a análise recai nas características individuais dos estudantes envolvidos em situações de bullying e nas relações entre pares (microssistema), especificamente.

No microssistema escola, nas relações entre pares, vão prevalecer comportamentos de amizade, cooperação, rivalidade ou agressão ${ }^{8,14}$. Quando as relações com os pares são afetadas por problemas de agressividade e violência, há um agravamento da violência e de 
Revista Baiana de Saúde Pública outros problemas individuais e coletivos ao longo do ciclo vital ${ }^{15}$. Esse tipo de compreensão contribui com a construção de modelos de intervenção intersetoriais fundamentados numa primeira etapa de definição e reconhecimento do problema e suas nuances no que se refere a protagonistas e formas de manifestação ${ }^{16}$.

Nesse sentido, este estudo objetivou identificar a prevalência e as características de vitimização por bullying entre estudantes brasileiros e portugueses de acordo com a idade, o sexo e o ano escolar, a partir das perspectivas da Teoria Bioecológica do Desenvolvimento.

\section{MATERIAL E MÉTODOS}

Neste estudo transversal participaram 182 estudantes (107 brasileiros e 75 portugueses). A amostra de conveniência foi composta por estudantes de duas escolas públicas, sendo uma do Brasil e uma de Portugal. Estabeleceu-se, como critério para inclusão dos estudantes: estar matriculado no $5^{0}$ ou no $6^{0}$ ano escolar; ser frequente às aulas; e estar presente nos dias de coleta de dados, que ocorreu no final do ano 2012 no Brasil e no início do ano de 2013 em Portugal. Destaca-se que o ano escolar em Portugal começa no mês de setembro e finaliza no mês de junho. Portanto, os períodos de coleta foram equivalentes, isto é, o segundo semestre letivo nos dois países.

Os comportamentos de bullying foram medidos por uma versão em português adaptada de um questionário ${ }^{17}$. Ele apresentava o conceito de bullying e estava estruturado em três blocos: Aspectos sociodemográficos (sexo, idade e dados escolares); Sobre ser ameaçado, humilhado ou agredido na escola; e Sobre ameaçar, maltratar, humilhar ou agredir alguém na escola. Este estudo privilegiou os dados das vítimas de bullying, assim são exemplos de questões do segundo bloco: Você já foi ameaçado, humilhado ou agredido na escola? O que fizeram com você? Em que lugar isso acontece ou aconteceu? Você contou para alguém quando isso aconteceu? Foram utilizadas duas versões do questionário, pois, mesmo sendo os grupos de estudantes de Língua Portuguesa, respeitaram-se os aspectos semânticos e linguísticos de cada uma das culturas investigadas. Essas versões foram adaptadas previamente para utilização em Portugal ${ }^{17}$ e para utilização no Brasil ${ }^{18}$.

No Brasil, uma escola pública do interior do estado de São Paulo foi selecionada por conveniência por constituir um campo de pesquisa e extensão universitária, vinculado ao grupo de pesquisa ao qual pertencem os pesquisadores. A coleta de dados foi realizada durante o período de aula dos estudantes, por meio de encontros com as turmas selecionadas ( $5 \underline{0}$ e $6^{\underline{o}}$ anos). Em Portugal, a pesquisa foi conduzida por uma equipe de Saúde Escolar que foi treinada pelos pesquisadores para a aplicação do questionário num agrupamento escolar público. 
A coleta de dados foi realizada durante o período de aula dos estudantes, por meio de encontros com as turmas selecionadas ( 5 e e 6 a anos). Tanto no Brasil quanto em Portugal, os pesquisadores apresentavam o questionário e, em seguida, permitiam o seu preenchimento, o que durava, em média, 30 minutos. Durante todo o procedimento os estudantes foram acompanhados e as dúvidas esclarecidas.

No programa Statistical Package for the Social Sciences (SPSS), primeiramente procedeu-se uma análise exploratória dos dados, com a intenção de verificar a existência de variações nos resultados. As respostas obtidas foram descritas em frequência e percentual. Nesse momento foram identificadas as vítimas com base no critério de ocorrência igual ou superior a três vezes nos últimos trinta dias. Posteriormente, utilizou-se o Teste Exato de Fisher para comparar as características apresentadas pelas vítimas dos dois países investigados. Adotou-se um nível de significância de $5 \%(p<0,05)$ para todas as análises. Os resultados foram organizados e descritos de duas maneiras: tabelas e medidas descritivas. A análise e a discussão foram referenciadas analiticamente pelos pressupostos da Teoria Bioecológica do Desenvolvimento 9 .

Os estudos foram aprovados, independentemente, em cada país. No Brasil foi aprovado pelo Comitê de Ética em Pesquisa da Escola de Enfermagem de Ribeirão Preto da Universidade de São Paulo (EERP-USP), protocolo 1.422/2011, parecer n. 471/2011. Em Portugal foi aprovado pela Subcomissão de Ética para as Ciências Sociais e Humanas da Direção-Geral da Educação (DGE) do Ministério da Educação e Ciência, protocolo 0101600013. As direções das duas escolas autorizaram a coleta de dados. No Brasil obteve-se também autorização da Diretoria Regional de Ensino de Ribeirão Preto. Os estudantes receberam informações detalhadas a respeito da pesquisa, para que pudessem decidir livremente sobre a sua participação e, para os interessados, disponibilizou-se o Termo de Consentimento Livre e Esclarecido, que deveria ser apresentado a um responsável e devolvido assinado aos pesquisadores. Para os estudantes brasileiros apresentou-se também o Termo de Assentimento, a ser assinado por eles mesmos, caso concordassem com a participação no estudo.

\section{RESULTADOS}

A amostra inicial foi composta por 182 estudantes, sendo 107 estudantes brasileiros e 75 portugueses. Após o levantamento, constatou-se que 40 sofriam bullying, dentre os quais $24,3 \%(n=26)$ eram do Brasil e 21,3\% $(n=16)$ de Portugal. A Tabela 1 apresenta as características sociodemográficas das 40 vítimas. Esse grupo compõe a amostra final deste estudo. 
Revista Baiana de Saúde Pública
Tabela 1 - Distribuição dos estudantes brasileiros e portugueses identificados como vítimas de bullying segundo sexo, idade, ano e reprovação escolar. Brasil 2012. Portugal -2013 . $(n=40)$

\begin{tabular}{|c|c|c|c|c|}
\hline \multirow{2}{*}{ Variáveis } & \multicolumn{2}{|c|}{ Brasil $(n=26)$} & \multicolumn{2}{|c|}{ Portugal $(n=14)$} \\
\hline & Frequência & $\%$ & Frequência & $\%$ \\
\hline \multicolumn{5}{|l|}{ Sexo } \\
\hline Feminino & 12 & 46,2 & 6 & 42,9 \\
\hline Masculino & 14 & 53,8 & 8 & 57,1 \\
\hline \multicolumn{5}{|l|}{ Idade } \\
\hline 10 & 1 & 3,8 & 2 & 14,3 \\
\hline 11 & 9 & 34,6 & 7 & 50,0 \\
\hline 12 & 9 & 34,6 & 5 & 35,7 \\
\hline 13 & 4 & 15,4 & - & - \\
\hline 15 & 3 & 11,6 & - & - \\
\hline \multicolumn{5}{|l|}{ Ano escolar } \\
\hline 5 & 14 & 53,8 & 6 & 42,9 \\
\hline 6 & 12 & 46,2 & 8 & 57,1 \\
\hline \multicolumn{5}{|l|}{ Reprovação } \\
\hline Sim & 6 & 23,1 & 3 & 21,4 \\
\hline Não & 20 & 76,9 & 11 & 78,6 \\
\hline
\end{tabular}

Fonte: Elaboração própria.

Nota: Sinal convencional utilizado:

- Dado numérico igual a zero não resultante de arredondamento.

No tocante ao sexo, identificou-se quantidade maior de estudantes vítimas do sexo masculino, porém não foram encontradas diferenças estatísticas significativas entre os sexos $(p=0,842)$. Os adolescentes da amostra brasileira apresentaram idade entre 10 e 15 anos, com média de 12,1 anos. Com uma média de idade menor, em torno de 11,2 anos, os adolescentes portugueses localizaram-se entre 10 e 12 anos. Apesar disso, não foi identificada diferença estatisticamente significativa em relação à idade dos participantes das duas amostras $(p=0,181)$. A distribuição dos participantes pelos dois anos escolares investigados mostrou-se equânime, com metade dos adolescentes em cada ano, quando analisados conjuntamente os dois países. Por outro lado, quando considerados separadamente, os estudantes brasileiros localizaram-se mais no $5^{\circ}$ ano e os portugueses mais no 6 o ano, diferença que não se mostrou significativa $(p=0,507)$. Em termos de proporção, o total de reprovações escolares foi equivalente dentre os dois grupos amostrais $(p=1,000)$ para Brasil e Portugal. 
Em relação ao tipo de agressão sofrida, os estudantes podiam assinalar mais de uma opção. A agressão verbal foi a mais sofrida nas duas amostras investigadas. Na amostra portuguesa figura uma concentração de agressões de natureza física e psicológica, ao passo que na amostra brasileira também se sobressaiu a quantidade de agressões de natureza física, além das agressões verbal e psicológica, conforme demonstrado na Tabela 2.

Tabela 2 - Distribuição das agressões sofridas segundo o tipo, local de ocorrência e se contou a alguém. Brasil - 2012. Portugal -2013. ( $n=40)$

\begin{tabular}{l|c|c|c|c}
\hline \multirow{2}{*}{ Variáveis } & \multicolumn{2}{|c|}{ Brasil (n=26) } & \multicolumn{2}{c}{ Portugal (n=14) } \\
\cline { 2 - 5 } & Frequência & $\%$ & Frequência & $\%$ \\
\hline Tipo de agressão & & & & \\
$\quad$ Física & 12 & 46,2 & 2 & 14,3 \\
$\quad$ Verbal & 21 & 80,8 & 8 & 57,1 \\
$\quad$ Psicológica & 13 & 50,0 & 7 & 50,0 \\
Local de ocorrência & & & & \\
$\quad$ Dentro da sala de aula & 4 & 15,4 & - & - \\
$\quad$ Fora da sala de aula & 22 & 84,6 & 14 & 100 \\
Contar a alguém & & & & \\
$\quad$ Não & 9 & 34,6 & 3 & 21,4 \\
$\quad$ Sim & 17 & 65,4 & 11 & 78,6 \\
A quem contou & & & & 33,3 \\
$\quad$ Professor & 20 & 42,6 & 7 & 33,3 \\
$\quad$ Pais & 11 & 23,4 & 7 & 23,8 \\
$\quad$ Amigos & 11 & 23,4 & 5 & 9,6 \\
$\quad$ Familiares & 5 & 10,6 & 2 &
\end{tabular}

Fonte: Elaboração própria.

Nota: Sinal convencional utilizado:

- Dado numérico igual a zero não resultante de arredondamento.

Malgrado as oscilações identificadas na frequência do tipo de agressão entre as duas amostras, não foram localizadas diferenças estatisticamente significativas para nenhuma delas: física $(p=0,177)$, verbal $(p=0,147)$ e psicológica $(p=1,000)$. No que tange ao local de ocorrência do bullying, a totalidade dos estudantes portugueses foi mais agredida fora da sala de aula, o mesmo ocorrendo com a maioria dos estudantes brasileiros. A similaridade nos resultados impediu a existência de diferença significativa $(p=0,278)$ entre os grupos. 
Revista Baiana de Saúde Pública
Identificou-se, ainda, outra convergência nos dois grupos amostrais investigados, no sentido da maioria dos estudantes brasileiros e portugueses relatarem a outras pessoas sobre sua condição de vítima ( $p=0,484)$. Dentre as pessoas para quem contam sobre as agressões que sofrem, destaca-se a figura do professor como sendo para quem os estudantes mais recorrem. Após os professores, os pais também são informados pelos adolescentes sobre o estado de vitimização em que se encontram. Igualmente contam para os amigos e, em proporção menor, para outros familiares. Nessa questão os estudantes podiam assinalar mais de uma opção.

\section{DISCUSSÃO}

Verificou-se, neste estudo, não haver diferenças entre os dois grupos amostrais de estudantes de Brasil e de Portugal no que se refere ao envolvimento com bullying na condição de vítima. Três fatores podem ajudar a explicar a ausência de diferenças entre os grupos: semelhança linguística, cultural e de interpretação sobre o conceito de bullying; similaridades na organização do sistema educacional, cujas políticas têm estimulado a discussão e a prevenção do bullying nas escolas, reservadas as particularidades macroestruturais dos dois países nesses aspectos; e ampla divulgação do fenômeno entre estudantes e professores nos dois países, bem como nos meios de comunicação.

Portanto, as semelhanças entre os dois países podem relacionar-se à proximidade na prevalência de bullying nos dois contextos investigados. De modo geral, a literatura científica sugere que diferenças contextuais e culturais explicam a variação de taxas de prevalência e níveis de envolvimento entre países ${ }^{19}$. Diferenças na organização do ensino e nas políticas educacionais entre os países, em geral, também justificam variações nas taxas de bullying entre dois grupos ${ }^{20}$. Além disso, o conhecimento da população sobre o bullying e a difusão de programas de intervenção nas escolas garante maior visibilidade ao fenômeno e, a longo prazo, a diminuição de ocorrências ${ }^{16,20}$. O que não se aplica ao presente estudo em função das semelhanças entre as duas realidades investigadas.

As taxas de prevalência de vitimização no Brasil (24,3\%) e em Portugal $(21,3 \%)$ foram elevadas e os meninos, como em outros estudos ${ }^{7,13}$, foram mais vítimas do que as meninas. Noutra direção, o elevado índice de não reprovação entre os estudantes das duas amostras indica que ele pode não ser um fator determinante para que o estudante sofra agressão no contexto escolar. Frente a esse cenário, cita-se um estudo que reuniu histórias de bullying e executou análises interpretativas de entrevistas, verificando que um adolescente que vivia sob ameaça e uma jovem identificada como tímida eram vítimas de bullying, mas não portavam histórias de reprovação ${ }^{21}$. 
Os atributos individuais, como o sexo, a idade e a trajetória escolar, determinam os recursos e habilidades que as pessoas utilizaram no processo de socialização para responder às demandas do contexto social ${ }^{13,22}$. No caso do bullying, as relações (processos proximais) entre os estudantes são caracterizadas como disruptivas para o desenvolvimento, marcadas por diferentes formas de manifestação. Neste estudo, verificou-se que a agressão verbal (apelidar, xingar, zoar, insultar) foi a mais sofrida nas duas amostras investigadas, estando presentes episódios de natureza física (bater, chutar, beliscar) e psicológica (intimidar, ameaçar, perseguir). As agressões ocorreram em sua maioria fora da sala de aula, o que é esperado, pois assim fica mais difícil para os adultos da escola presenciarem e, consequentemente, intervirem para finalizar as agressões ${ }^{1,21}$.

Como as relações entre pares determinam como as pessoas vão socializar-se, interagir e comportar-se socialmente, a qualidade dessas relações representa risco ou proteção para o bullying ${ }^{8,10}$. Interações inadequadas podem prejudicar a saúde e o desenvolvimento dos estudantes, bem como impactar no processo ensino-aprendizagem. Em outras palavras, a violência presente nas relações determina o fluxo e as condições de saúde e de desenvolvimento ${ }^{12}$. Esse é o núcleo mais contextual abordado pelo estudo, pois abrange o ciclo de relações mais próximas dos estudantes, sendo uma das dimensões em que ocorrem os processos de desenvolvimento. Nessas relações, em geral, as vítimas de bullying colocam-se como passivas, frágeis, tímidas e sem recursos internos para se defenderem dos agressores. Ao mesmo tempo, as escolas que interpretam os episódios de bullying como brincadeiras ou como problemas de mau comportamento de responsabilidade da família, impedem que sejam fomentadas relações positivas entre os estudantes, baseadas na tolerância às diferenças e à diversidade $\mathrm{e}^{9,22-23}$.

Noutra direção, tanto os estudantes brasileiros quanto os portugueses referiram ter contado a outras pessoas sobre sua condição de vítima, sinalizando que eles não tendem a sofrer sozinhos, solicitando o apoio de outras pessoas que podem auxiliá-los a deixarem a condição de vítima. O professor foi a pessoa a quem os estudantes mais recorreram. Em geral, os professores são considerados pelos estudantes como os que mais possuem possibilidades de Ihes auxiliar em situações de bullying. Entretanto, percebe-se que, em muitos casos, os professores não se encontram preparados ou não sabem como enfrentar o problema ${ }^{24}$.

Essa abordagem permite compreender o bullying enquanto um importante determinante para a saúde, o desenvolvimento e o processo ensino-aprendizagem de crianças e adolescentes. Ao mesmo tempo, identificam-se os efeitos diretos e indiretos dos componentes individuais e micro dos sistemas ecológicos que, sobrepostos e relacionados aos outros níveis, interferem no processo de vitimização dos estudantes. Na análise nas vítimas de bullying, em 
Revista Baiana de Saúde Pública resultados de dois grupos de indivíduos de realidades sociais diferentes, pode-se pensar em conexões teóricas e práticas que focalizem respostas de contexto e coletivas. Nesse sentido, especificamente na área da saúde e na capilaridade dos territórios nos dois países, podem ser pensadas ações intersetoriais.

No Brasil são estimuladas ações de promoção de saúde no contexto escolar que contemplem, de forma transversal, a temática do bullying ${ }^{25-26}$. Essas iniciativas devem ser desenvolvidas no âmbito da atenção primária e podem compreender ações que estimulem relações sociais positivas e saudáveis entre todos os estudantes, bem como contribuir com os professores e demais funcionários para reconhecer e lidar com o bullying. De forma efetiva, o Programa Saúde na Escola (PSE) aproxima no cotidiano as equipes de saúde e escolar e favorece a construção de canais de interlocução para a abordagem do tema, a prevenção da violência e o desenvolvimento de novas e saudáveis possibilidades de resolução de conflitos ${ }^{26}$.

Em Portugal, a efetividade dos serviços de Saúde Escolar, as parcerias com universidades e outros setores da sociedade apresentam boas experiências. Naquele país, o trabalho de diagnóstico de situações de bullying e a adoção de estratégias de enfrentamento contemplam diferentes perspectivas ${ }^{17,21}$. As vítimas e as testemunhas de situações de bullying são estimuladas a relatarem os episódios de violência, os professores e funcionários das escolas são treinados para identificar agressões e intervir de forma eficaz e o foco é a construção de ambientes seguros e que favoreçam a saúde e o desenvolvimento de crianças e adolescentes em idade escolar ${ }^{17,21}$. Consensualmente, o bullying, explicado como resultado de uma ampla gama de fatores que compõem a ecologia social, requer ações complexas e multiníveis em seu enfrentamento.

\section{CONSIDERAÇÕES FINAIS}

Este estudo identificou a prevalência e as características de vitimização por bullying entre estudantes brasileiros e portugueses, por meio de perspectivas do desenvolvimento. Não foram identificadas diferenças significativas entre os dois grupos amostrais. A Teoria Bioecológica do Desenvolvimento contribuiu para a compreensão da complexidade do fenômeno e pode auxiliar na construção de estratégias antibullying em escolas brasileiras e portuguesas. Os pontos fortes deste estudo referem-se às contribuições para diferentes áreas, como a educação e a saúde, enquanto setores que devem promover ações de cuidado e atenção às crianças e aos adolescentes. Ao mesmo tempo, a compreensão do fenômeno em países lusófonos pode contribuir para o aumento de pesquisas em parceria e dos esforços para combater e prevenir o bullying escolar entre estudantes de língua portuguesa. 
Entretanto, as contribuições deste estudo devem ser interpretadas considerando algumas limitações. Primeiramente, utilizaram-se apenas estudantes como informantes e as pesquisas sugerem que outros informantes devem ser incluídos em pesquisas sobre bullying, como os professores, por exemplo. Essa medida ampliaria o olhar sobre o fenômeno e como outros atores o interpretam, vivenciam ou significam no contexto escolar. Além disso, embora o uso de questionários represente um método adequado para a coleta de dados sobre o bullying, ele possui limitações relacionadas a dificuldades de compreensão e/ou mesmo de reconhecimento dos episódios de agressão. Outra limitação é que nenhuma informação coletada relacionava o bullying a questões do desenvolvimento, saúde ou do processo ensino aprendizagem explicitamente. No entanto, o plano interpretativo desenvolvido pelos pesquisadores analisou os dados por meio dessas dimensões, minimizando vieses de tal natureza. A limitação final do estudo refere-se ao seu delineamento transversal que impede inferências sobre nexos causais entre as variáveis estudadas.

Apesar das limitações apresentadas, este estudo abre caminho para pesquisas qualitativas ou com delineamento misto que podem auxiliar na compreensão da experiência e dos sentidos atribuídos pelos estudantes ao bullying. Investigações com foco nos estudantes identificados como agressores também são estimuladas, uma vez que existem lacunas na literatura científica nessa perspectiva. Sugere-se, ainda, pesquisar sobre as reais e possíveis consequências do bullying para a saúde, o desenvolvimento e processo ensino-aprendizagem dos estudantes.

\section{AGRADECIMENTOS}

Os autores agradecem à Fundação de Amparo à Pesquisa do Estado de São Paulo (FAPESP) pela concessão de bolsas de doutorado para o primeiro e o segundo autor (processos 2014/13062-7 e 2013/22361-5, respectivamente). Agradecem, também, ao Conselho Nacional de Desenvolvimento Científico e Tecnológico (CNPq) por conceder bolsa de doutorado para a terceira autora.

\section{COLABORADORES}

1. Concepção do projeto, análise e interpretação dos dados: Wanderlei Abadio de Oliveira, Jorge Luiz da Silva, Julliane Messias Cordeiro Sampaio, Beatriz Oliveira Pereira e Marta Angélica lossi Silva

2. Redação do artigo e revisão crítica relevante do conteúdo intelectual: Jorge Luiz da Silva. 
Revista Baiana de Saúde Pública
3. Revisão e/ou aprovação final da versão a ser publicada: Marta Angélica lossi Silva.

4. Ser responsável por todos os aspectos do trabalho na garantia da exatidão e integridade de qualquer parte da obra: Wanderlei Abadio de Oliveira.

\section{REFERÊNCIAS}

1. Olweus D. School bullying: development and some important challenges. Annu Rev Clin Psychol. 2013;9(1):751-80.

2. Oliveira WA, Silva MAI, Silva JL, Mello FCM, Prado RR, Malta DC. Associations between the practice of bullying and individual and contextual variables from the aggressors' perspective. J Pediatr (Rio J). 2016;92:32-9.

3. Zequinão MA, Cardoso AA, Silva JL, Medeiros P, Silva MAI, Pereira B, et al. Academic performance and bullying in socially vulnerable students. J Hum Growth Dev. 2017;27(1):19-27.

4. Smith CR, Fisher BS, Gillespie GL, Beery TA, Gates DM. Adolescents' experience with workplace aggression: school health implications. J Sch Nurs. 2013;29(6):464-74.

5. Cook CR, Williams KR, Guerra NG, Kim TE. Variability in the prevalence of bullying and victimization: A cross-national and methodological analysis. In: Jimerson SR, Swearer S, Espelage DL, editors. Handbook of bullying in schools: An international perspective. New York: Routledge/Taylor \& Francis Group; 2010. p. 347-62.

6. Silva MAI, Pereira B, Mendonca D, Nunes B, Oliveira WA. The involvement of girls and boys with bullying: an analysis of gender differences. Int J Environ Res Public Health. 2013;10(12):6820-31.

7. Rech R, Halpern R, Tedesco A, Santos DF. Prevalence and characteristics of victims and perpetrators of bullying. J Pediatr (Rio J). 2013;89(2):164-70.

8. Silva JL, Oliveira WA, Braga IF, Farias MS, Lizzi EAS, Fagundes MG, et al. The effects of a skill-based intervention for victims of bullying in Brazil. Int J Environ Res Public Health. 2016;13:1042-52.

9. Bronfenbrenner U. A ecologia do desenvolvimento humano: tornando os seres humanos mais humanos. Porto Alegre: ArtMed; 2011.

10. Patton DU, Hong JS, Williams AB, Allen-Meares P. A review of research on school bullying among African American youth: an ecological systems analysis. Educ Psychol Rev. 2013;25(2):245-60. 
11. Hong JS, Peguero AA, Choi S, Lanesskog D, Espelage DL, Lee NY. Social Ecology of bullying and peer victimization of Latino and Asian youth in the United States: a review of the literature. J Sch Violence. 2013;13(3):315-38.

12. Lee $\mathrm{CH}$, Song J. Functions of parental involvement and effects of school climate on bullying behaviors among South Korean Middle School students. J Interpers Violence. 2012;27(12):2437-64.

13. Schultz NCW, Duque DF, Silva CF, Souza CD, Assini LC, Carneiro MGM. A compreensão sistêmica do bullying. Psicol estud. 2012;17:247-54.

14. Mendes CS. Prevenção da violência escolar: avaliação de um programa de intervenção. Rev esc enferm USP. 2011;45:581-8.

15. Ortega-Ruiz R, Nunez JC. Bullying and cyberbullying: Research and intervention at school and social contexts. Psicothema. 2012;24(4):603-7.

16. Silva JL, Oliveira WA, Mello FCM, Andrade LS, Bazon MR, Silva MAI. Revisão sistemática da literatura sobre intervenções antibullying em escolas. Ciênc Saúde Colet. 2017;22(7):2329-40.

17. Melim FM. Na escola, tu és feliz? Estudo sobre as manifestações e implicações do bullying escolar [tese]. Braga: Universidade do Minho; 2012. 459 p.

18. Barros PC. Jogos e brincadeiras na escola: prevenção do bullying entre crianças no recreio [tese]. Braga: Universidade do Minho; 2012. 217 p.

19. Sentenac M, Gavin A, Arnaud C, Molcho M, Godeau E, Nic Gabhainn S. Victims of bullying among students with a disability or chronic illness and their peers: a cross-national study between Ireland and France. J Adolesc Health. 2011;48(5):461-6.

20. Kouwenberg M, Rieffe C, Theunissen S, Rooij M. Peer ictimization experienced by children and adolescents who are deaf or hard of hearing. PLoS One. 2012;7(12):e52174.

21. Ferraz $S$, Pereira B. Comportamentos de bullying: estudo numa escola técnico profissional. In: Condessa I, Pereira B, Carvalho C, editors. Atividade física, saúde e lazer. Educar e formar. Braga: Centro de Investigação em Estudos da Criança, Instituto de Educação; 2012. p. 93-9.

22. Freire AN, Aires JS. A contribuição da psicologia escolar na prevenção e no enfrentamento do Bullying. Psicol Esc Educ. 2012;16:55-60.

23. Silva JL, Oliveira WA, Bazon MR, Cecilio S. Bullying na sala de aula: percepção e intervenção de professores. Arq bras psicol. 2013; 65(1):121-37.

24. Silva JL, Oliveira WA, Bazon MR, Cecilio S. Bullying: conhecimentos, atitudes e crenças de professores. Psico. 2014;45(2):147-56. 
Revista Baiana de Saúde Pública
25. Brasil. Ministério da Saúde. Secretaria de Atenção à Saúde. Departamento de Ações Programáticas e Estratégicas. Linha de cuidado para a atenção integral à saúde de crianças, adolescentes e suas famílias em situação de violência. Brasília; 2010.

26. Ferreira IRC, Moysés SJ, França BHS, Carvalho ML, Moysés ST. Percepções de gestores locais sobre a intersetorialidade no Programa Saúde na Escola. Rev Bras Educ. 2014;19:61-76.

Recebido: 10.3.2016. Aprovado: 17.11.2017. Publicado: 6.7.2018. 